\title{
Identification aveugle de filtres ARMA et applications
}

\author{
M. BOUMAHDI
}

CEPHAG/ENSIEG, Domaine Universitaire, BP. 46, 38402 Saint-Martin-d'Hères cedex, France

\begin{abstract}
The blind identification of linear systems is an important problem in signal processing (communication, seismic,..). The second order statistics (Autocorrelation function, or Power Spectral Density), are blind phase, they only permit the identification of minimum phase linear time invariant systems. During the past years there has been an increasing interest in utilizing the Higher Order Statistics (HOS), to solve the problem of non-minimum phase blind identification. Because the HOS keep information about the phase. To identify ARMA filters with unknowns orders, we use all the information given by the second order statistics, and complete it by searching to maximize the kurtosis. We apply this approach to field seismic data.
\end{abstract}

\section{INTRODUCTION}

En acoustique, l'onde émise est souvent assimilée à un filtre linéaire ; l'identification aveugle consiste à estimer la fonction de transfert de ce filtre, uniquement à partir des statistiques du signal enregistré sur un capteur, en supposant que l'entrée du fïltre est un bruit blanc et que la sortie correspond au signal enregistré. Identifier l'onde émise revient donc à identifier le filtre.

L'approche classique d'identification aveugle consistant à n'utiliser que les statistiques d'ordre deux (Autocorrélation ou densité spectrale de puissance), ne permet que l'identification des filtres à phase minimale. Une nouvelle approche permettant d'identifier les filtres quelles que soient leurs phases, et qui s'est développée récemment, consiste à utiliser les statistiques d'ordre supérieur à deux.

Notre approche est d'assimiler l'onde émise à un processus $\operatorname{ARMA}(p, q)$ réel et causal, et de l'identifier en utilisant toute l'information de l'ordre deux, et la compléter par une information donnée par les statistiques d'ordre quatre (Le Kurtosis). D'abord nous testons cette approche sur des signaux simulés, puis nous l'utilisons pour traiter des données réelles de sismique sous-marine

\section{DÉFINITIONS}

2.1 Statistiques d'ordre quatre d'un processus aléatoire

Soit $\{\mathrm{x}(\mathrm{t})\}$ un processus aléatoire centré réel, discret et stationnaire d'ordre quatre, sa tricorrélation est:

$$
\mathrm{K}_{\mathrm{X}}(1, \mathrm{~m}, \mathrm{n})=\mathrm{E}\{\mathrm{x}(\mathrm{t}) \mathrm{x}(\mathrm{t}+\mathrm{l}) \mathrm{x}(\mathrm{t}+\mathrm{m}) \mathrm{x}(\mathrm{t}+\mathrm{n})\}-\Gamma_{\mathrm{X}}(1) \Gamma_{\mathrm{X}}(\mathrm{m}-\mathrm{n})-\Gamma_{\mathrm{X}}(\mathrm{m}) \Gamma_{\mathrm{X}}(1-\mathrm{n})-\Gamma_{\mathrm{X}}(\mathrm{n}) \Gamma_{\mathrm{X}}(1-\mathrm{m})
$$

Où $\Gamma_{x}(1)=E\{x(t) x(t+1)\}$ est l'autncorrélation de $x(t)$ à l'instant 'l'.

Le trispectre de $\mathrm{x}(\mathrm{t})$ est défini comme la transformée de Fourier discrète tridimensionelle (TFD3D) de la tricorrélation:

$$
\mathrm{T}_{\mathrm{x}}\left(\mathrm{v}_{1}, \mathrm{v}_{2}, \mathrm{v}_{3}\right)=\mathrm{TFD} 3 \mathrm{D}\left[\mathrm{K}_{\mathrm{x}}(1, \mathrm{~m}, \mathrm{n})\right]
$$

Le kurtosis est égal à la valeur de la tricorrélation à l'origine, normalisée par le carré de la puissance:

$$
\operatorname{Kurt}[x(t)]=\left[K_{x}(0,0,0)\right] /\left[\Gamma_{x}(0)\right]^{2}
$$

Sa valeur indique "la distance" qui sépare la densité de probabilité de $\mathrm{x}(\mathrm{t})$ de la gaussiéneté. 


\subsection{Statistiques de la sortie d'un système linéaire}

Soit $\mathrm{x}(\mathrm{t})$ la sortie d'un filtre linéaire de réponse impulsionelle $\mathrm{h}(\mathrm{t})$ invariante dans le temps, et dont l'entrée est excitée par $\mathrm{r}(\mathrm{t})$, un bruit blanc d'ordie quatre [1]:

$$
x(t)=\sum_{i=0}^{\infty} h(i) r(t-i)
$$

L'équation de Brillinger-Rosenblatt [2] donne:

1) à l'ordre quatre:

$$
\mathrm{K}_{\mathbf{X}}(1, \mathrm{~m}, \mathrm{n})=\zeta \sum_{\mathbf{i}} \mathrm{h}(\mathrm{i}) \mathrm{h}(\mathrm{i}+1) \mathrm{h}(\mathrm{i}+\mathrm{m}) \mathrm{h}(\mathrm{i}+\mathrm{n}) \quad \text { et } \mathrm{T}_{\mathrm{X}}\left(\mathrm{v}_{1}, \mathrm{v}_{2}, \mathrm{v}_{3}\right)=\zeta \mathrm{H}\left(\mathrm{v}_{1}\right) \mathrm{H}\left(\mathrm{v}_{2}\right) \mathrm{H}_{(}\left(\mathrm{v}_{3}\right) \mathrm{H}^{*}\left(\mathrm{v}_{1}+\mathrm{v}_{2}+\mathrm{v}_{3}\right)
$$

2) à l'ordre deux:

$$
\Gamma_{\mathrm{X}}(\tau)=\alpha \sum_{\mathrm{i}} \mathrm{h}(\mathrm{i}) \mathrm{h}(\mathrm{i}+\tau) \quad \text { et } \quad \gamma_{\mathrm{X}}(\mathrm{v})=|\mathrm{H}(\mathrm{v})|^{2} \alpha
$$

Avec $\mathrm{K}_{\mathrm{r}}(1, \mathrm{~m}, \mathrm{n})=\zeta \delta(1, \mathrm{~m}, \mathrm{n}), \Gamma_{\mathrm{r}}(\tau)=\alpha \delta(\tau), \delta($.$) est la fonction de Kronecker, \mathrm{H}(\mathrm{v})$ est la Transformée de Fourier Discrète de $h(t)$, et $\gamma_{x}(v)$ est la densité sepectrale de $x(t)$.

L'ordre deux (5) donne une information sur le module du gain complexe, et supprime toute information sur sa phase ; il ne permet d'estimer que les phases minimales à partir du module [3]. Au contraire, l'ordre quatre (6) apporte une information sur le module et sur la phase du gain complexe du filtre.

\subsection{Kurtosis de la sortie d'un filtre linéaire}

Le kurtosis de $\mathrm{x}(\mathrm{t})$ s'exprime en fonction du kurtosis de $\mathrm{r}(\mathrm{t})$ et de la réponse impulsionelle $\mathrm{h}(\mathrm{t})$ [4]:

$$
\operatorname{kurt}[\mathrm{x}(\mathrm{t})]=\operatorname{kurt}[\mathrm{r}(\mathrm{t})]\left[\sum_{\mathrm{i}} \mathrm{h}^{4}(\mathrm{i})\right]\left[\sum_{i} \mathrm{~h}^{2}(\mathrm{i})\right]^{2} \leq \operatorname{kurt}[\mathrm{r}(\mathrm{t})]
$$

Cette relation montre que le kurtosis de $x(t)$ est plus petit que celui de $r(t)$. Celà signifie que $x(t)$ est "plus gaussien" que $r(t)$ [4]. Soit $f(t)$ la réponse impulsionelle d'un filtre linéaire invariant dans le temps, qui filtrant $x(t)$ donne $u(t)$. Le kurtosis de $u(t)$ est:

$$
\operatorname{kurt}[u(t)]=\operatorname{kurt}[r(t)]\left[\sum_{i} g^{4}(i)\right]\left[\sum_{i} g^{2}(i)\right]^{2} \leq \operatorname{kurt}[r(t)] \quad \text { avec } g(i)=h(i) * f(i)
$$

Le kurtosis de $r(t)$ est la borne supérieure, des kurtosis de toutes les filtrées possibles de $\mathrm{x}(\mathrm{t})$. Une procédure de déconvolution consiste à chercher le filtre $\mathrm{f}(\mathrm{t})$ qui filtrant, $\mathrm{x}(\mathrm{t})$ donne un signal $\mathrm{u}(\mathrm{t})$ ayant un kurtosis maximum. Le maximum des kurtosis sera obtenu lorsque $u(t)$ est identique à $r(t)$ [4], c'est à dire lorsque:

$$
f(t) * h(t)=c \delta\left(t-t_{0}\right) \text {, avec c et } t_{0} \text { constantes }
$$

Dans la suite de cet article nous utiliserons la maximisation du kurtosis comme critère d'identification de filtres ARMA à phase non-minimale.

\section{IDENTIFICATION DE FILTRES ARMA}

Soit le processus $\operatorname{ARMA}(p, q)$, réel et causal, décrit par l'équation aux différences suivante:

$$
x(t)+\sum_{i=1}^{p} a(i) x(t-i)=r(t)+\sum_{i=1}^{q}(i) r(t-i)
$$

La fonction de transfert en $Z$, associée à ce processus est: $H(z)=\sum_{i} h(i) z^{-i}=\left[1+\sum_{i=1}^{q} b(i) z^{-i}\right]\left[1+\sum_{i=1}^{p} a(i) z^{-i}\right]$

Avec:

- $x(t)$ est la sortie du filtre. $r(t)$ est un bruit blanc d'ordre quatre [1], (entree non-accessible du filtre). $h(t)$ est la réponse impulsionelle du filtre.

- $\{a\}_{i=1}^{p}$ et $\{b\}_{i=1}^{q}$ sont respectivement les coefficients de la partie AR et de la partie MA.

Comme le filtre est causal, tous les pôles de sa (TZ) sont à l'intérieur du cercle unité. L'information sur la phase (minimale ou non) est contenue dans la localisation des zéros de la partie MA(q). Si tous les zéros sont à l'intérieur du cercle unité celà signifie que le filtre est à phase minimale. Sinon le filtre est à phase non-minimale

\subsection{Identification de la partie $A R$}


La partie AR de l'ARMA peut être identifiée à l'ordre deux en utilisant l'équation de Yule-Walker [3]. A condition toutefois qui'il n'y ait pas de facteurs passe-tout (allpass factors) dans H(z) [1]. Car ces facteurs sont "invisibles" à l'ordre deux. Une solution à ce problème consiste à étendre l'équation de Yule-Walker aux statistiques d'ordre supérieur [1]:

$$
\mathrm{K}_{\mathrm{X}}(\mathrm{k}, \mathrm{n}, 0)=-\sum_{\mathrm{i}=1}^{\mathrm{p}} \mathrm{a}_{\mathrm{i}} \mathrm{K}_{\mathrm{x}}(\mathrm{k}-\mathrm{i}, \mathrm{n}, 0) \quad \text { pour } \mathrm{l} \geq \mathrm{q}+1 \text { et } \mathrm{n}=\mathrm{q}-\mathrm{p}, \ldots, \mathrm{q}
$$

\subsection{Identification de la partie MA}

Le signal observé $\mathrm{x}(\mathrm{t})$ est filltré par $\hat{\mathrm{A}}(\mathrm{z})$ estimé dans (3.1), le réultat est un signal $\mathrm{u}(\mathrm{t})$ ne contenant que l'information sur la partie MA(q):

$$
u(t)=r(t)+\sum_{i=1}^{q} b_{i} r(t-i)
$$

Si la TZ de la partie MA(q) contient des zéros à l'éxtérieur du crecle unité. Elle peut s'écrire :

$$
B(z)=\prod_{i=1}^{q_{1}}\left(1-\sigma_{i} z^{-1}\right) \prod_{j=1}^{q_{2}}\left(1-\alpha_{j} z^{-1}\right) \quad \text { et } \quad B_{P M}(z)=\prod_{i=1}^{q_{1}}\left(1-\sigma_{i} z^{-1}\right) \prod_{j=1}^{q_{2}}\left(1-\left(1 / \alpha_{j}^{*}\right) z^{-1}\right)
$$

avec $q_{1}+q_{2}=q,\left|\sigma_{i}\right|<1,\left|\alpha_{j}\right|>1$ et $B_{P M}(z)$ est le filtre à phase minimale spectralement équivalent.

$\mathrm{B}_{\mathrm{PM}}(\mathrm{z})$ est identifiable à l'ordre deux [3]. Tous ses zéros sont à l'intérieur du cercle unité. Pour passer de $\mathrm{B}_{\mathrm{PM}}(\mathrm{z})$ à $\mathrm{B}(\mathrm{z})$, il suffit de chercher les zéros qu'il faut remplacer par leurs inverses conjugués. Chaque inversion de zéros définit un filtre $\mathrm{B}^{\mathrm{i}}(\mathrm{z})$, chacun d'eux en déconvoluant $\mathrm{u}(\mathrm{t})$ donne un bruit blanc d'ordre deux $\mathrm{r}^{\mathrm{i}}(\mathrm{t})$. $\mathrm{B}(\mathrm{z})$ correpond au filtre donnant $\mathrm{r}^{\mathrm{i}}(\mathrm{t})$ ayant le maximum des kurtosis. Cette condition exprime la blancheur d'ordre quatre de $\mathrm{r}^{\mathrm{i} O}(\mathrm{t})[4][5][6]$.

\subsection{Determination de l'ordre d'un ARMA}

Pour estimer l'ordre du filtre, nous proposons de chercher le filtre ARMA(k,n) donnant le maxixmum du kurtosis. Pour $\mathrm{k}=1, \ldots, \mathrm{p}, \ldots \mathrm{p}_{\max }$, et $\mathrm{n}=1, \ldots \ldots \mathrm{q}, . . \mathrm{q}_{\max }$.

\section{CAS DE LA SISMIQUE REFLEXION}

En sismique, $h(t)$ correspond à la signature de l'onde émise dans le sous-sol (Ondelette). $r(t)$ correspond au signal réflectivité qui contient l'information sur la position des réflecteurs du sous-sol, et $\mathrm{x}(\mathrm{t})$ (Trace sismique) correspond au signal enregistré sur un capteur, il est considéré comme le résultat de la convolution de $r(t)$ par $h(t)$. Le but de la déconvolution est de localiser les positions et l'amplitude des reflecteurs, afin d'identifier la composition des couches.

\subsection{Simulation}

La réflectivite $r(t)$ est simulée par une série de fonctions de Kronecker, d'amplitudes et de positions temporelles aléatoires. L'ondelette $h(t)$ est un filtre $\operatorname{ARMA}(6,5)$, dont nous supposons l'ordre inconnu. Sa TZ est $H(z)=\left(1-0.13 z^{-1}-2.26 z^{-2}+4.13 z^{-3}-0.68 z^{-4}+2.32 z^{-5}\right) /\left(1-1.4 z^{-1}+1.2 z^{-2}-0.9 z^{-3}+0.7 z^{-4}-0.5 z^{-5}+0.4 z^{-6}\right)$

$\mathrm{La}$ trace sismique obtenue est le résultat de la convolution de $\mathrm{r}(\mathrm{t})$ par $\mathrm{h}(\mathrm{t})$.

Pour $\mathrm{k}=1, \ldots, 10$ et $\mathrm{n}=1, \ldots, 8$, nous cherchons le filtre $\operatorname{ARMA}(\mathrm{k}, \mathrm{n})$ donnant le maximum du kurtosis (voir Tab 1).

Le maximum des kurtosis est obtenu pour un filtre $\operatorname{ARMA}(6,5)$ donnant un kurtosis de 26.44 , et dont la (TZ) est $\hat{H}(z)=\left(1-0.18 z^{-1}-1.97 z^{-2}+3.72 z^{-3}-0.66 z^{-4}+2.23 z^{-5}\right) /\left(1-1.38 z^{-1}+1.19 z^{-2}-0.89 z^{-3}+0.68 z^{-4}-0.47 z^{-5}+0.37 z^{-6}\right)$

Cet exemple montre la capacité de l'approche utilisée d'identifier des filtres ARMA d'ordre inconnu.

\subsection{Traitement de données réelles}

Pour traiter des données sismiques réelles, il faut se mettre dans le cadre des hypothèses: l'ondelette est invariante dans le temps, il y a un bon rapport signal sur bruit, et seulement un seul type d'onde doit exister. Pour être dans ces conditions, les données sont d'abord traités par la martice spectrale [7]. 
Les données que nous traitons nous ont été fournies par $\mathrm{Mr}$ P. Charvis de l'O.R.S.T.O.M. Nous en présentons le résultat obtenu sur 16 traces de 128 points chacune.

(Fig 1) représente les traces avant déconvolution, (Fig 2) représente le résultat de la déconvolution par maximisation du kurtosis, et (Fig 3) représente le résultat de la déconvolution par l'ondelette à phase minimale.

La déconvolution par maximisation du kurtosis permet de mieux localiser les reflecteurs, et met en évidence de façon plus nette trois arrivées sismiques. L'inversion de signe entre la première et la deuxième arrivée, est identifiée comme étant un mutilple.

\section{CONCLUSION}

Le résultat obtenu sur les données réelles prouve l'applicabilité des statistiques d'ordre supérieur, à des données expérimentales pour résoudre le problème de déconvolution aveugle à phase non-minimale en sismique. Toutefois ce résultat n'aurait pu être obtenu si on avait directement travaillé sur les données.

L'utilisation de la matrice spectrale a permis d'être plus proche du modèle théorique adopté. Cela nous conduit à conclure que le problème de la résolution du problème de la déconvolution aveugle, en sismique ne peut être résolu qu'en utilisant un prétraitement de séparation d'ondes sismiques.

Tab 1: Valeurs des kurtosis

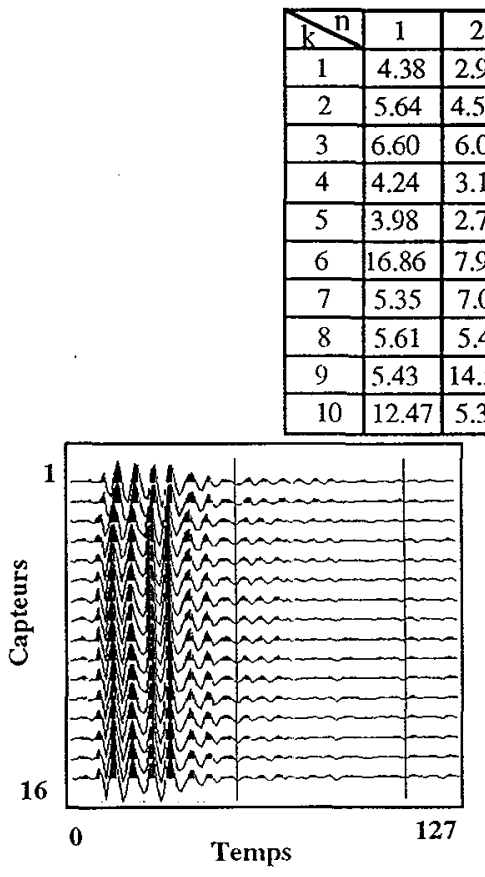

Fig 1

\section{Références:}

Fig 2

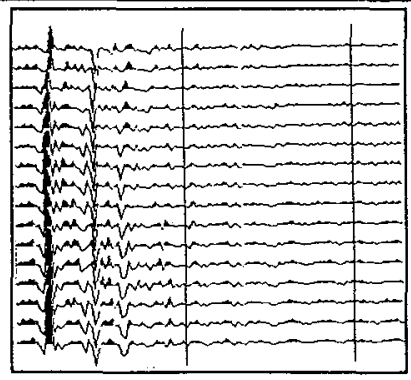

Fig 3

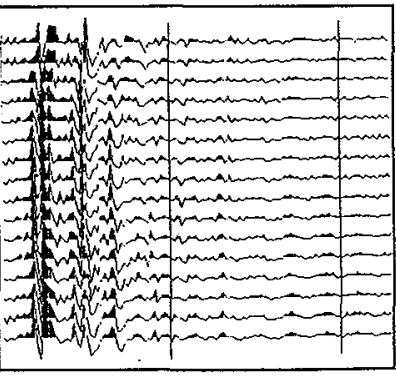

[1] J. Mendel, "Tutorial on Higher-Order Statistics (Spectra) in Signal Processing ans system Theory: Theorical Results and Some Applications", Procedings of the IEEE, vol. 79, no 3, March 1991.

[2] D.R Brillinger and M. Rosenblatt, "Computation and interpretation of kth-order spectra", in spectral analysis of time series, B. Harris ed. New York: Wiley, pp.189-232, 1967.

[3] Steven M. Kay. "Modern Spectral Estimation, Theory and Applications". Prentice-Hall Signal Processing Series, Alan. Oppenheim, Series Editor

[4] D.L Donoho "On minimum entropy deconvolution" 556-608, in applied time series analysis II, D.F. Findley, (ed), Academic press.

[5] M. Boumahdi, F. Glangeaud \& J.L. Lacoume, "Deconvolution aveugle en sismique utilisant les statistiques d'ordre supérieur", GRETSI 93, 13-16 Septembre, Juan les Pins, France.

[6] M. Boumahdi, "Intérêt des statistiques d'ordre supérieur pour la déconvolution aveugle", 4éme C.M.M.N.I, 22-24 Novembre 1993, Alger, Algerie.

[7] J. Mars, F. Glangeaud, J.L. Lacoume, I.M. Founnann, and S. Spitz, "Séparation of Seismic Waves" Proceding of S.E.G, New Orleans, Oct 1987. 